\title{
Perfil de resistência de agentes de micoses oportunistas no Brasil
}

Resistance profile of opportunistic mycose agents in Brazil

Jamile Cristine dos Santos MAGALHÃES'

Geisiany Maria de QUEIROZ-FERNANDES²*

\section{RESUMEN}

O uso indiscriminado de antifúngicos vem contribuindo com a mudança no perfil epidemiológico de fungos resistentes, elevando a taxa de mortalidade nos pacientes acometidos. No Brasil, devido à ausência de dados oficiais, não existem relatos precisos sobre a primeira detecção de resistência a antifúngicos. Entretanto, dentre os agentes causadores de micoses oportunistas a espécie mais isolada é a Candida albicans, além de outras espécies como Cryptococcus neoformans, Sporothrix schenckii e Aspergillus spp. têm sido descritas. Este estudo buscou evidenciar o progressivo aumento dos índices de resistência de fungos oportunistas aos antifúngicos comumente utilizados e ressaltar a importância do conhecimento do perfil de sensibilidade antes do início do tratamento, pois o tratamento incorreto destas infecções agrava as falhas terapêuticas e leva à remissão das micoses. Realizou-se revisão de literatura científica com base no banco de dados Bireme, Scielo, Pubmed e Google Acadêmico, utilizando como descritores: Candida albicans, Sporothrix schenckii, Cryptococcus neoformans, Aspergillus spp., micoses oportunistas, resistência, resistência em micoses oportunistas, perfil de resistência e resistência fúngica no Brasil. Os mesmos foram pesquisados em português, inglês e espanhol. Com o crescente número de pacientes imunocomprometidos os índices de infecções oportunistas aumentaram. Patógenos fúngicos oportunistas, pertencentes a estes gêneros, mostraram-se resistentes aos poliênicos (anfotericina B), imidazóis (cetoconazol، miconazol, econazol), flucitosina, triazóis (voriconazol, fluconazol, itraconazol) e multiazóis em muitos estudos. A utilização inadequada de alguns antifúngicos vem contribuindo para o aumento de taxas de incidência de infecções oportunistas, principalmente por cepas resistentes, piorando o prognóstico destes pacientes.

Palavras-chave: Antifúngicos; Aspergillus spp.; Candida albicans; Cryptococcus neoformans; Sporothrix schenckii.

\section{ABSTRACT}

The indiscriminate use of antifungals has contributed to the change in the epidemiological profile of resistant fungi, increasing the mortality rate in affected patients. In Brazil, due to the absence of official data, there are no precise reports on the first detection of antifungal resistance. Among the causative agents of opportunistic mycoses, the most isolated species is Candida albicans, but other species such as Cryptococcus neoformans, Sporothrix schenckii and Aspergillus spp. have been described. This study aimed to highlight the progressive increase in resistance rates of opportunistic fungi to commonly used antifungals and to highlight the importance of knowing the sensitivity profile before the start of treatment, because the incorrect treatment of these infections aggravates therapeutic failures and leads to the remission of mycoses. $A$ review of scientific literature was conducted based on the Bireme, Scielo, Pubmed and Google Acadêmico database, using with descriptors: Candida albicans, Sporothrix schenckii, Cryptococcus neoformans, Aspergillus spp., opportunistic mycoses, resistance, resistance in opportunistic mycoses, resistance profile and fungal resistance in Brazil. They were searched in Portuguese, English and Spanish. With the increasing number of immunocompromised patients, the rates of opportunistic infections have increased. Opportunistic fungal pathogens belonging to these genera have been shown to be resistant to polyenes (amphotericin B), imidazole (ketoconazole, miconazole, econazole), flucitosin, triazole (voriconazole, fluconazole, itraconazole) and multiazoles in many studies. The inadequate use of some antifungals has contributed to the increase in incidence rates of opportunistic infections, especially by resistant strains, worsening the prognosis of these patients.

Keywords: Antifungals; Aspergillus spp.; Candida albicans; Cryptococcus neoformans; Sporothrix schenckii.

${ }^{1}$ Centro Universitário Unisagrado. Bauru, SP, Brasil.

${ }^{2}$ Faculdade São Leopoldo Mandic de Araras, Curso de Medicina. Av. Dona Renata, 71, 13600-001, Centro, Araras, SP, Brasil. Correspondência para: GM QUEIROZ- FERNANDES. E-mail: <geisiany.fernandes@slmandicararas.edu.br>.

Como citar este artigo / How to cite this article

Magalhães JCS, Queiroz-Fernandes GM. Perfil de resistência de agentes de micoses oportunistas no Brasil. InterAm J Med Health 2021;4:e202101010. https://doi.org/10.31005/iajmh.v4i.149 


\section{INTRODUÇÃO}

Georgopoulos, em 1969[1], alertou que problemas relacionados à resistência a fungicidas deveriam se tornar mais frequentes e severos no mundo, especialmente, devido à utilização de novos antifúngicos mais seletivos do que os convencionais. Tal fato se concretizou, pois desde então é possível observar o aumento do número de relatos de resistência a fungicidas. Em 1997, em Manchester, na Inglaterra, o primeiro caso de resistência a itraconazol, em isolado clínico de Aspergillus fumigatus, foi relatado[2]. No Brasil, não existem relatos precisos sobre a primeira detecção de resistência a antifúngicos, devido à ausência de dados oficiais de notificação[3].

Entretanto, nota-se que atualmente existe uma constante mudança no quadro epidemiológico de diferentes formas de resistência fúngica, pois o uso indiscriminado de alguns antifúngicos contribui para a seleção natural de cepas resistentes, elevando a taxa de mortalidade dos pacientes acometidos [4-7]. Esses micro-organismos desenvolveram diversos mecanismos para evitar a ação dos antifúngicos como, por exemplo, alteração do sítio de ação e da permeabilidade [6].

As infecções causadas fungos oportunistas vêm aumentando em frequência e importância nos últimos anos, principalmente em pacientes imunocomprometidos e dentre os agentes causadores de micoses oportunistas, a espécie mais isolada é a Candida albicans, porém outras espécies como Cryptococcus neoformans, Sporothrix schenckii e Aspergillus spp. também têm sido descritas $[4,5,8,10]$.

Na última década, as infecções causadas por outras espécies de Candida não albicans e também resistentes têm aumentado muito. A resistência intrínseca observada em algumas espécies de Candida e o desenvolvimento de resistência adquirida durante o tratamento têm dificultado o controle de candidíases $[10,11]$.

Em meados do século $X X$, devido ao aumento de indivíduos imunocomprometidos, a incidência da criptococose aumentou significadamente [12]. Devido ao uso prolongado de fluconazol, fármaco antifúngico triazólico que possui excelente penetração no sistema nervoso central (SNC), existe a preocupação com o surgimento de resistência antifúngica em $\mathrm{C}$. neoformans [13].

Dentre as doenças com potencial zoonótico, a esporotricose é reconhecida como uma micose emergente com distribuição mundial, que tende a aumentar exponencialmente, e tem sido relatada no Brasil desde 1907 $[14,15]$. Apesar de o tratamento com drogas antifúngicas ser eficaz, estudos demonstraram o surgimento de cepas de Sporothrix spp. resistentes, incluindo o itraconazol, fármaco de escolha para o tratamento $[5,15,17]$.

A aspergilose, micose oportunista provocada por fungos do gênero Aspergillus, tem constituído um problema grave, sobretudo, em indivíduos imunossuprimidos, pois espécies pertencentes a este gênero podem adquirir resistência a múltiplas terapias fúngicas [18,19].

Devido ao aumento das taxas de seleção de cepas fúngicas resistentes, principalmente entre os agentes de micoses oportunistas, este estudo teve como objetivos evidenciar as mudanças no perfil de resistência fúngica, no Brasil e ressaltar a importância do conhecimento do perfil de sensibilidade destes micro-organismos antes do início do tratamento.

\section{MATERIAL E MÉTODOS}

Para o desenvolvimento deste estudo, realizouse revisão de literatura, sendo organizada nas seguintes etapas: definição do problema e implantação da pergunta norteadora de pesquisa; estabelecimento das palavraschave; organização da estratégia de busca a ser inserida nas plataformas de dados e determinação dos critérios de inclusão e exclusão dos artigos; seleção dos artigos; determinação das informações a serem extraídas dos resultados obtidos, metodologia, resultados e conclusão e interpretação dos resultados encontrados.

Elaborou-se como pergunta norteadora a seguinte questão: "Houve mudança no quadro epidemiológico de micoses oportunistas, no Brasil, devido à seleção de cepas resistentes a agentes antifúngicos?". Para a seleção dos trabalhos estabeleceu-se como critério de inclusão artigos e/ou dissertações com temática pertencente à mudança do perfil de resistência de agentes de micoses oportunistas e/ou características sobre os fungos, sendo consideradas publicações nacionais e internacionais, dando preferência para estudos dos últimos cinco anos (2014 a 2019), publicados nas plataformas Bireme, Scielo, Pubmed e Google Acadêmico. Excluíram-se publicações que, mesmo citando os fungos foco deste estudo, não tratavam sobre o tema proposto.

Para a realização da busca foram utilizadas as palavras-chave: Candida albicans, Sporothrix schenckii, Cryptococcus neoformans, Aspergillus spp., micoses oportunistas, resistência, resistência em micoses 
oportunistas, perfil de resistência e resistência fúngica no Brasil. As palavras-chave foram pesquisadas em português, inglês e espanhol.

Para a seleção, realizou-se a leitura de cada título, resumo e introdução com a finalidade de verificar se os estudos se enquadravam aos critérios de inclusão e exclusão. Após a seleção, realizou-se a leitura completa para a elaboração deste trabalho. Foram encontrados um total de 95 estudos, sendo que 53 foram selecionados e incluídos na elaboração deste artigo.

\section{Candida albicans}

C. albicans é uma levedura que coloniza de forma assintomática diversas partes do corpo, estando mais concentrada nos tratos gastrintestinal e geniturinário, porém na presença de condições favoráveis, a mesma pode se tornar patogênica e causar infecções que podem variar desde uma manifestação local até quadros sistêmicos que podem levar o indivíduo a óbito $[4,9,11,20,21]$. Essa levedura é capaz de expressar vários fatores de virulência como adesinas, alteração da sua morfogênese, secreção de enzimas, formação de biofilmes, entre outros [21,22]. $O$ processo infeccioso causado pelos fungos do gênero Candida, conhecido como candidíase ou candidose, varia de acordo com o sítio anatômico e em geral pode ser classificado em três grupos: mucocutâneo, que atinge mucosa oral e vaginal, cutâneo e sistêmico, quando ocorre o comprometimento de vários órgãos e/ou sistemas [21].

Com o crescente número de pacientes imunocomprometidos, devido às infecções virais, doenças, transplantes, linfomas, terapêutica imunodepressiva, envelhecimento, dentre outras razões, e com a seleção de espécies com reduzida suscetibilidade aos antifúngicos, notaram-se alterações nos índices epidemiológicos de infecções causadas por Candida, nos últimos anos. Leveduras resistentes aos poliênicos (nistatina e anfotericina B) têm sido relatadas $[23,24]$. Para o fluconazol, antifúngico sistêmico que se mostrava eficaz contra a maioria das espécies de Candida, hoje observam-se diferentes graus de sensibilidade. O surgimento de resistência ao fluconazol tem sido descrito especialmente em C. albicans, C. tropicalis e C. parapsilosis [25]. Diversos estudos relataram que o tratamento prévio com antibacterianos e fluconazol é um fator de risco para candidemia, podendo selecionar cepas de Candida não albicans resistentes. Este aumento da resistência tem sido observado em quadros de candidemia causada por C. glabrata e C. krusei após tratamento prolongado com vancomicina e piperacilina-tazobactam [26].
Um estudo realizado entre 2002 e 2003, em Porto Alegre, visava determinar a distribuição de espécies de Candida associadas à candidemia e seus perfis de sensibilidade antifúngica à anfotericina $\mathrm{B}$, fluconazol e itraconazol, através do método de microdiluição em caldo. Os autores observaram presença de sensibilidade dosedependente ao itraconazol em isolados de C. krusei (50\%), C. glabrata $(25 \%)$, C. parapsilosis $(16 \%)$, C. tropicalis $(12 \%)$ e C. albicans (7\%) e ao fluconazol foi encontrada somente para os isolados de C. krusei [24].

Outro estudo realizado com indivíduos infectados pelo Vírus da Imunodeficiência Humana (HIV) atendidos nos serviços de saúde de Cuiabá e Várzea Grande- Mato Grosso, no período entre 2006 e 2008, com o objetivo de caracterizar leveduras do gênero Candida de distintas amostras clínicas e determinar o perfil de sensibilidade in vitro a cinco antifúngicos. Em relação ao perfil geral de sensibilidade, um isolado $C$. krusei demonstrou resistência para cetoconazol, fluconazol, itraconazol e anfotericina B, exceto ao voriconazol. Ao analisar os 82 isolados de $C$. albicans, foram observadas altas CIM, indicando resistência de C. albicans, sendo estes valores iguais a $29,6 \%$ (fluconazol), 30,8\% (itraconazol), 25,9\% (voriconazol), 23,4\% (cetoconazol) e 1,2\% para a anfotericina B [27].

Considerando todos os antifúngicos avaliados, $O$ fluconazol foi aquele que apresentou menor ação, ou seja, apresentou maiores valores de CIM, sugerindo resistência para a maioria dos isolados, sendo C. krusei (100\%), C. albicans (29,6\%), C. tropicalis (50\%). Também foi observado um número elevado de isolados resistentes ao itraconazol e cetoconazol, para as espécies: C. krusei $(75 \%)$, C. albicans $(30,8 \%)$, C. tropicalis $(50 \%), C$. krusei $(50 \%)$, C. albicans $(23,4 \%)$ e C. tropicalis $(37,5 \%)$ respectivamente. A espécie $C$. parapsilosis mostrou-se resistente ao itraconazol (25\%) e anfotericina B (12,5\%). Também foram encontrados isolados resistentes a anfotericina $\mathrm{B}$, com destaque para os isolados de C. krusei (75\%) [27].

Goulart et al. (2018) [11] investigaram a sensibilidade a antifúngicos de espécies de Candida isoladas de pacientes HIV positivo, em Mato Grosso, em 2015. Estes autores notaram que os testes de sensibilidade revelaram que $84 \%$ dos isolados de Candida spp., 1\% apresentou resistência ao fluconazol e ao cetoconazol e $4 \%$ ao itraconazol.

Em estudo realizado entre 2010 e 2011 com pacientes internados em um hospital de Uberlândia/MG, observaram que dos 63 isolados, houve resistência in vitro à anfotericina B em 12,7\% e aos azólicos para os isolados de C. krusei. A resistência à anfotericina $B$ foi observada 
em um isolado de C. albicans, em um do complexo de C. parapsilosis, e em seis outras espécies, entre C. krusei, C. glabrata e C. kefyr [28].

Com o objetivo de avaliar a resistência de C. tropicalis isoladas no ano de 2008 de amostras clínicas no Ceará, 51 amostras de $C$. tropicalis foram avaliadas frente a drogas padrões: anfotericina B, fluconazol e itraconazol. Foram detectadas três $(5,9 \%)$ cepas de $C$. tropicalis resistentes ao fluconazol e três $(5,9 \%)$ cepas de $C$. tropicalis resistentes ao itraconazol [29].

Nogueira et al. (2008) [30] isolaram, identificaram e determinaram a sensibilidade antimicrobiana dos microorganismos causadores de otite externa de 27 pacientes. Dentre as leveduras causadoras desta condição foram isoladas uma cepa de C. albicans e uma cepa de C. krusei, ambas foram sensíveis à anfotericina $B$, nistatina, fluconazol e clotrimazol e resistentes ao miconazol.

Em um estudo realizado em um hospital de referência da região norte do Brasil, em Belém (PA), entre 2008 e 2010, foram isoladas 81 leveduras do gênero Candida, sendo $40,7 \%$ pertencentes à espécie albicans e $25,9 \%$ a espécie tropicalis. Os isolados de C. tropicalis foram resistentes dose-dependente ao itraconazol. A resistência ao fluconazol foi observada $3,7 \%$ das cepas e ao voriconazol 4,9\%. Das Candida spp 16\% foram resistentes ao itraconazol e todas as leveduras foram resistentes ao fluconazol, voriconazol e ao itraconazol [31].

Silva et al. (1998) [32] coletaram amostras da mucosa oral de 86 pacientes com Síndrome da Imunodeficiência Adquirida (AIDS) atendidos em um hospital em Goiânia de onde foram isoladas cepas de diferentes espécies de Candida spp., os testes de sensibilidade mostraram resistência de $25,42 \%$ ao itraconazol e $45,76 \%$ ao cetoconazol. A maior taxa de resistência foi observada para o fluconazol $(66,10 \%)$. Todos os isolados de C. krusei foram resistentes ao fluconazol, enquanto $75 \%$ dos isolados de C. tropicalis foram também resistentes a este fármaco.

Durante um estudo desenvolvido em 2008, em Jundiaí, 13 pacientes apresentaram infecções hospitalares por Candida spp., sendo que cinco desses pacientes estavam na Unidade de Terapia Intensiva (UTI) e oito na Unidade de Terapia Intensiva Pediátrica (UTIP). Sete dos 13 pacientes apresentaram infecção por C. albicans; três por C. tropicalis, um por C. parapsilosis, um por C. krusei e um por C. guilliermondii. Três pacientes foram a óbito, sendo que todos os isolados de Candida spp. foram sensíveis à anfotericina $B$, nistatina e fluconazol. Em relação ao econazol e itraconazol, algumas apresentaram sensibilidade intermediária e uma cepa de C. albicans foi resistente ao econazol. Dois dos pacientes que faleceram eram recémnascidos imunossuprimidos. O terceiro paciente era uma criança de sete anos que teve co-morbidade cardíaca e foi internado na UTIP [33].

\section{Cryptococcus neoformans}

Cryptococcus neoformans é uma levedura encapsulada, ubíqua, encontrada em todo mundo, responsável por aproximadamente um milhão de casos de infecções por ano [13,34]. A manifestação clínica, conhecida como criptococose, é sistêmica com evolução subaguda ou crônica, sendo o comprometimento da imunidade celular o principal fator predisponente para a infecção. Ao ocorrer a inalação dos propágulos leveduriformes, esses atingem os pulmões e pode ocorrer disseminação hematogênica para o cérebro e meninges, visto que este fungo tem predileção pelo SNC $[35,36]$. Possui diferentes fatores de virulência como a própria cápsula polissacarídica e a produção de melanina, que ao ser sintetizada intracelularmente, é transferida para a parede celular, tornando as células de $C$. neoformans menos suscetíveis a antifúngicos e fagocitose [36].

Mesmo com os avanços no desenvolvimento de drogas antifúngicas, houve um aumento de infecções por Cryptococcus spp. que não responderam ao fluconazol, fármaco de primeira escolha. Atualmente, os fármacos de primeira escolha para a terapia a longo prazo ainda são os derivados azólicos, porém o surgimento de isolados de C. neoformans e C. gattii resistentes ao fluconazol, representam um grande desafio. O uso indiscriminado de antifúngicos tem contribuído para a seleção de cepas resistentes a estes agentes, especialmente, em pacientes com AIDS que desenvolvem criptococose $[37,38]$.

Em um estudo realizado entre 1990 e 2004 com 1811 isolados clínicos de C. neoformans, que visava determinar a tendência global de sensibilidade antifúngica, no qual participaram países da África, América Latina, Europa, Ásia e América do Norte, notaram-se que somente 75\% dos isolados da América do Norte foram sensíveis a fluconazol, comparados a 94 e $100 \%$ em outras regiões. A sensibilidade à flucitosina ocorreu somente em $35 \%$ dos isolados na América do Norte e em 68\% na América Latina [37].

Com o imunocomprometimento causado pela infecção pelo HIV e a disponibilidade de antifúngicos, o conceito relacionado à cura e a recaída da criptococose tem sido alterado. Normalmente, os pacientes são tratados 
com anfotericina B com ou sem flucitosina como terapia inicial, porém a terapia de manutenção é necessária em pacientes com AIDS com infecções por C. neoformans. Em um relato de caso ocorrido com um paciente com AIDS que apresentou resistência durante a terapia de manutenção com fluconazol, apresentando recidiva da meningite criptocócica, a CIM para fluconazol, que no primeiro episódio de meningite era $<8 \mathrm{ug} / \mathrm{mL}$, elevou-se para $64 \mathrm{ug} / \mathrm{mL}$, demonstrando redução da sensibilidade ao medicamento [37,39].

Em estudo que visava analisar a sensibilidade de 53 cepas de C. neoformans de isolados clínicos e ambientais, os autores observaram cepas resistentes ao fluconazol e que estas cepas resistentes foram mais frequentes do que o esperado [40].

Outro estudo realizado em Mato Grosso no período de 2005 a 2008, avaliou 37 isolados de dez pacientes HIV negativos e 26 positivos, e observou-se que os isolados clínicos C. neoformans de HIV positivos apresentaram resistência entre $8 \%$ e $8,7 \%$ para fluconazol e sensibilidade dose-dependente de $20 \%$ e $17,4 \%$ para itraconazol [41]. A baixa sensibilidade dos isolados ao fluconazol vem sendo relatada com grande frequência, pois também no Rio de Janeiro, verificou-se resistência a este antifúngico em $75,86 \%$ dos isolados de C. neoformans obtidos de fezes de pombos [42].

Silva et al. (2008) [43] avaliaram a sensibilidade a antifúngicos em isolados de 35 pacientes do Hospital Escola da Universidade Federal do Triângulo Mineiro e detectaram-se dois isolados resistentes ao itraconazol e dois a anfotericina $B$, sendo um da variedade gattii e o outro da variedade neoformans.

\section{Sporothrix schenckii}

Sporothrix schenckii é um fungo dimórfico que causa micose subcutânea, conhecida como esporotricose. A esporotricose é uma infecção causada por uma das espécies do complexo Sporothrix que é composto por pelo menos seis espécies crípticas (S. schenckii, S. brasiliensis, S. globosa, S. mexicana, S. luriei e S. albicans). A inoculação do fungo na área subcutânea deve-se a uma lesão traumática com a fonte de infecção, que normalmente é ocasionada por partículas de vegetais ou espinhos, visto que este fungo é comumente encontrado em plantas em decomposição e no solo. Além disso, a esporotricose tem sido associada a arranhões e/ou mordidas de animais silvestres e raramente por inalação dos esporos $[5,14,44$, 45]. Esta infecção se apresenta como lesões e/ou nódulos cutâneos subagudos ou crônicos, ulcerado ou verrucoso, com ou sem disseminação linfática local, podendo ocorrer disseminação para vários órgãos e sistemas. Nos casos mais graves podem ocorrer lesões oculares, pulmonares, infecção generalizada, entre outros $[5,14,46]$. Acredita-se que os fatores de virulência têm relação com a produção de proteinases extracelulares produzidas pelo fungo, que hidrolisam a elastina e o colágeno [46].

O tratamento da esporotricose normalmente é realizado com itraconazol, sendo este considerado o padrão ouro para as formas cutâneas e linfocutâneas da micose em humanos. Entretanto, estudos relataram a existência de isolados resistentes a este fármaco, levando consequentemente a falhas terapêuticas e disseminação da micose $[15,16,47]$.

Em um estudo realizado com objetivo de determinar a atividade in vitro da terbinafina e itraconazol frente a 12 isolados clínicos de S. schenckii, sendo seis casos de esporotricose felina, cinco de esporotricose humana e um proveniente de micose em cão, foram observadas resistências ao itraconazol no isolado de cão e nos demais isolados provenientes de esporotricose felina [16].

Oliveira et al. (2015) [47] avaliaram a sensibilidade in vitro de S. albicans, S. brasiliensis, S. globosa, S. mexicana e S. schenckii à terbinafina em combinação com itraconazol, cetoconazol e voriconazol e observaram que todos os isolados de S. schenckii e S. brasiliensis foram sensíveis ao itraconazol, porém S. albicans, S. globosa e S. mexicana mostraram resistência às combinações, sendo que a mais relevante delas foi observada em ralação ao voriconazol.

Em outro estudo realizado no Sul do Brasil, com isolados de S. schenckii e S. brasiliensis de humanos, gatos, cães, solo e uma estirpe padrão (IOC 1226) para a determinação da atividade antifúngica de produtos naturais. Observaram-se que cinco isolados de gatos e um de cão foram resistentes ao antifúngico comercial utilizado como controle, o itraconazol, especialmente, S. brasiliensis [15].

\section{Aspergillus spp.}

Este gênero fúngico é composto por centenas de espécies saprofíticas, cada uma com distintas características morfológicas, macroscópicas e microscópicas, sendo amplamente distribuídas na natureza. Cerca de uma dezena dessas espécies são capazes de provocar infecção no homem e em animais. Destaca-se o Aspergillus fumigatus como a espécie mais frequentemente isolada, sendo responsável por $90 \%$ das infecções em humanos. Outras espécies como A. flavus, A. nidulans, A. terreus e A. niger são consideradas agentes de infecções relativamente 
frequentes $[16,48]$. A exposição a estes fungos no meio ambiente pode provocar reações alérgicas em doentes hipersensíveis ou aspergilose invasiva e doença disseminada em indivíduos com imunocomprometimento [18].

A aspergilose é uma infecção fúngica oportunista, tendo como via primária o trato respiratório, a partir da inalação dos conídios anemófilos infectantes. Estudos recentes sugerem que a água pode também ser uma fonte de infecção, devido à grande capacidade de esporulação que estes fungos possuem em meio aquoso, sendo capazes de formar micronichos ambientais [49]. Com a inalação dos esporos, o quadro pode variar de doenças pulmonares, que vão desde a inflamação local das vias aéreas até infecções graves com risco de vida. Isto ocorre porque os esporos ao serem inalados, germinam, e dão origem a hifas nos pulmões, que tendem a invadir também outros tecidos e/ ou órgãos [18].

Durante algum tempo, o fenômeno de resistência adquirida por parte da aplicação dos antifúngicos azólicos no tratamento de Aspergillus spp. foi desconhecido. Porém esta evidência e prevalência têm evoluído nas últimas décadas, resultando na ineficácia no combate do patogênico A. fumigatus, principalmente. A resistência ao itraconazol por Aspergillus spp. é conhecida desde meados de 1990 e a resistência a triazóis por A. fumigatus foi descrita em 1997, e em 1999, surge o relato da ocorrência de resistência a multiazóis[19]. Outra espécie de grande relevância, A. terreus, traz grande preocupação devido à resistência à anfotericina B [18]. Cepas de Aspergillus spp. possuem resistência cruzada a outros fármacos e fungicidas azólicos, e esse fato compromete o tratamento de pacientes com aspergilose bronco pulmonar alérgica e/ou outras formas de aspergilose [50].

Nos últimos anos a resistência aos azólicos em isolados de Aspegillus spp. foi rigorosamente investigada e diferentes mecanismos foram descritos, tanto observados antes da exposição ao triazóis, quanto adquiridos durante a terapia. Particularmente na Europa, Ásia e Brasil, a utilização de azólicos na agricultura tem sido descrita como a causa do surgimento de isolados de A. fumigatus resistentes a triazóis [18,51,52].

Em um estudo realizado entre 2001 a 2017 pela Universidade de São Paulo em Ribeirão Preto, foram analisados o perfil de sensibilidade de 32 isolados clínicos de Aspergillus spp. obtidos de amostras clínicas de diferentes sítios anatômicos, frente a anfotericina B, itraconazol, voriconazol e posaconazol. Os achados deste estudo mostraram que isolados de A. fumigatus apresentaram resistência ao itraconazol, posaconazol e voriconazol. E que um isolado de A. brasiliensis apresentou resistência a itraconazol [53].

\section{CONCLUSÃO}

Micoses oportunistas são infecções frequentes entre os pacientes imunocomprometidos, causando graves problemas de saúde púbica. A utilização inadequada de alguns antifúngicos vem contribuindo para o aumento da seleção e incidência de cepas resistentes. O tratamento incorreto destas infecções agrava as falhas terapêuticas e remissão das micoses.

Neste contexto, o presente estudo revelou que há um aumento progressivo no perfil de resistência de fungos oportunistas frente a diferentes agentes antifúngicos, no Brasil, sendo, portanto, de suma importância o conhecimento do perfil de sensibilidade destes fungos antes do início do tratamento, para que haja melhoria no prognóstico do paciente.

\section{REFERÊNCIAS}

1. Georgopoulos SG. The problem of fungicide resistance. Bioscience. 1969;19(11): 971-973. http://dx.doi. org/10.2307/1294971

2. Denning DW, Radford SA, Oakley $K L$, Hall $L$, Johnson EM, Warnock DW. Correlation between invitro susceptibility testing to itraconazole and invivo outcome of Aspergillus fumigatus infection. J Antimicrob Chemother. 1997;40(3):401-14. http://dx.doi. org/10.1093/jac/40.3.401

3. Mesquita-Rocha S. Aspergillus fumigatus: aspectos gerais e importância na medicina contemporânea. J Health Sci Inst. 2019;37(2):169-73.

4. Macalupú SZ. Situación de la resistência antifúngica de espécies del gênero Candida en Perú. Rev Peru Med Exp Salud Publica. 2018;35(1): 126-31. http://dx.doi. org/10.17843/rpmesp.2018.351.3563.

5. Guirado GMP, Feitosa LS, Kozusny-Andreani DI, Navarro RS. Analysis of laser therapy effects in Sporothrix schenckii inactivation in vivo. Res Biomed Eng. 2018;34(2): 93-101. http://dx.doi.org/10.1590/2446-4740.06917

6. Garcia A. A resistência dos fungos como consequência da utilização de fungicidas sistêmicos: mecanismos de 
resistência, monitoramento e estratégias anti-resistência. Porto Velho: Embrada Rondônia; 1999.

7. Agência Fundação de Amparo à Pesquisa do Estado de São Paulo- Agência FAPESP. Agência. Resistência fúngica. [acesso 2018 Ago 26]. Disponível em: http://agencia. fapesp.br/resistencia-fungica/6284/.

8. Alburquenque OC, Silva AV, Fuentes GM, Tapia CC, Silva VV. Susceptibilidad in vitro a anidulafungina em 100 cepas de especies de Candida aisladas previo a la introducción de esta equinocandina en Chile. Rev Chil Infect. 2011; 28(5): 399-403. http://dx.doi.org/10.4067/ S0716-10182011000600001

9. Quintana SC, Sjostrom PD, Baldeón GM, Socarrás DA, Paz MC, Molina AH. Genoma de Candida albicans y resistencia a las drogas. Salud Uninorte Barranquilla. 2017;33(3): 438-50.

9. Robledo-Leal E, Rivera-Morales LG, Sangorrín MP, González GM, Ramos-Alfano G, Adame-Rodriguez JM, et al. Identification and susceptibility of clinical isolates of Candida spp. to killer toxins. Braz J Biol. 2018;78(4): 74249. http://dx.doi.org/10.1590/1519-6984.175635.

10. Goulart LS, Souza WWR, Vieira CA, Lima JS, Olinda RA, Araújo C. Oral colonization by Candida species in HIVpositive patients: association and antifungal susceptibility study. Einstein (São Paulo). 2018;16(3)1-6. http://dx.doi. org/10.1590/S1679-45082018A04224

11. Cattana ME, Tracogna MF, Fernández MS, CarolRey MC, Sosa MA, Giusiano GE. Genotipificación de aislamientos clínicos del complejo Cryptococcus neoformans/Cryptococcus gattii obtenidos em el Hospital Dr. Julio C. Perrando, de la ciudad de Resistencia (Chaco, Argentina). Rev Argent Microbiol. 2013;45(2): 89-92. http://dx.doi.org/10.1016/S0325-7541(13)70005-1

12. Bosco-Borgeat ME, Mazza M, Taverna CG, Córdoba $S$, Murisengo OA, Vivot $W$, et al. Amino acid substitution in Cryptococcus neoformans lanosterol 14- $₫$-demethylase involved in fluconazole resistance in clinical isolates. Rev Argent Microbiol. 2016;48(2):137-42. http://dx.doi. org/10.1016/j.ram.2016.03.003

13. Ferreira LC, Barroso PF, Tonomura E, Akiti T, Rodrigues
KMP. Osteomyelitis caused by Sporothrix schenckii in an immunocompetent patient. Rev Soc Bras Med Trop. 2016;49(4):527-29. http://dx.doi.org/10.1590/00378682-0354-2015

14. Waller SB, Madrid IM, Cleff MB, Satin R, Freitag RA, Meireles MCA, et al. Effects of essential oils of Rosmarinus officinalis Linn. and Origanum vulgare Linn. from different origins on Sporothrix brasiliensis and Sporothrix schenckii complex. Arq Bras Med Vet Zootec. 2016;68(4):991-99. http://dx.doi.org/10.1590/1678-4162-8962

15. Meinerz ARM, Nascente PS, Schuch LFD, Cleff MB, Santin R, Brum CS, et al. Suscetibilidade in vitro de isolados de Sporothrix schenckii frente à terbinafina e itraconazol. Rev da Soc Bras de Med Trop. 2007;40(1):60-2. http:// dx.doi.org/10.1590/S0037-86822007000100012

16. Waller SB, Madrid IM, Faria RO, Cleff MB, Mello JRB, Meireles MCA. Anti-Sporothrix spp. activity of medicinal plants. Braz J of Pharm Sci. 2016;52(2): 221-38. http:// dx.doi.org/10.1590/S1984-82502016000200001

17. Carvalho LIC. Aspergillus e aspegilose- desafios no combate da doença [dissertação]. Porto: Universidade Fernando Pessoa; 2013.

18. Valente JRS. Estudo molecular de estirpes de Aspergillus fumigatus isolados em aviários e em aves diagnosticadas com espergilose. Possíveis implicações na Saúde Pública [dissertação]. Portugal: Faculdade de Ciências e Tecnologia- Universidade Nova de Lisboa; 2014.

19. Souza CES, Leite NF, Brito DIV, Alenca LBB, Lavor AKLS, Matias EFF, et al. Avaliação da atividade antimicrobiana e moduladora do extrato hexânico de Costus cf. Arabicus L. Rev Cubana de Plant Med. 2014;19(2):151-59.

20. Melo IA, Guerra RC. Candidíase oral: um enfoque sobre a estomatite por prótese. Salusvita. 2014;33(3):389414.

21. Calderone RA, Fonzi WA. Virulence factors of Candida albicans. Trends in Microbiology. 2001;9(7):327-335. http://dx.doi.org/10.1016/S0966-842X(01)02094-7

22. Alves SH, Cury AE. Sensibilidade de leveduras do gênero Candida, isoladas de pacientes com câncer, a antifúngicos 
poliênicos. Rev Inst Med Trop S Paulo. 1992;34(3):251-54. http://dx.doi.org/10.1590/S0036-46651992000300012

23. Antunes AGV, Pasqualotto AC, Diaz MC, Azevedo PA Severo LC. Candidemia in a Brazilian tertiary care hospital: species distribution and antifungal susceptibility patterns. Rev Inst Med Trop S Paulo. 2004;46(5):239-41. http:// dx.doi.org/10.1590/S0036-46652004000500001

24. Furlaneto MC, Rota JF, Quesada RMB, Furlaneto-Maia $L$, Rodrigues $R$, Oda $S$, et al. Species distribution and in vitro fluconazole susceptibility of clinical Candida isolates in a Brazilian tertiary-care hospital over a 3-year period. Rev Soc Bras Med Trop. 2011;44(5):595-99. http://dx.doi. org/10.1590/S0037-86822011000500013

25. Leite GML, Baeza LC, Ramos RT, Yamada SS, Magon TFS, Kimura E, et al. Lack of effect of cell-wall targeted antibacterials on biofilm formation and antigungal susceptibility of Candida species. Braz J of Pharm Sci. 2014;50(3): 467-72. http://dx.doi.org/10.1590/S198482502014000300004

26. Favalessa OC, Martins MA, Hahn RC. Aspectos micológicos e suscetibilidade in vitro de leveduras do gênero Candida em pacientes HIV-positivos provenientes do Estado de Mato Grosso. Rev Soc Bras Med Trop. 2010;43(6): 673-77. http://dx.doi.org/10.1590/S003786822010000600014

27. Menezes RP, Ferreira JC, Sá WM, Moreira TA, Malvino LDS, Araujo LB, et al. Frequency of Candida species in a tertiary care hospital in Triangulo Mineiro, Minas Gerais State, Brazil. Rev Inst Med Trop. 2015;57(3): 185-91. http://dx.doi.org/10.1590/S0036-46652015000300001

28. Menezes EA, Mendes LG, Cunha FA. Resistência a antifúngicos de Candida tropicalis isoladas no Estado do Ceará. Rev Soc Bras Med Trop. 2009;42(3):354-55. http:// dx.doi.org/10.1590/S0037-86822009000300024

29. Nogueira JCR, Diniz MFFM, Lima EO, Lima ZN. Identificação e susceptibilidade antimicrobiana de microrganismos obtidos de otite externa aguda. Rev Bras Otorrinolaringol. 2008;74(4):526-30. http://dx.doi. org/10.1590/S0034-72992008000400007

30. Nunes EB, Nunes NB, Monteiro JCMS, Paes ALV. Perfil de sensibilidade do gênero Candida a antifúngicos em um hospital de referência da Região Norte do Brasil. Rev PanAmaz Saude. 2011;2(4):23-30. http://dx.doi.org/10.5123/ S2176-62232011000400004

31. Silva MRR, Paula CR, Silva SC, Costa TR, Costa MR. Drug resistance of yeasts isolated from oropharyngeal candidiasis in aids patients. Rev Microbiol. 1998;29(4):271-75. http:// dx.doi.org/10.1590/S0001-37141998000400007

32. Storti LR, Pasquale G, Scomparim R, Galastri AL, Alterthum F, Gambale W, et al. Candida spp. isolated from inpatients, the environment, and health practitioners in the pediatric unit at the Universitary Hospital of the Jundiaí Medical College, state of São Paulo, Brazil. Rev Soc Bras Med Trop. 2012;45(2):225-31. http://dx.doi.org/10.1590/ S0037-86822012000200017

33. Atiya $Y$, Masege SD. Cryptococcal laryngitis: An uncommon presentation of a common pathogen. S Afr Med J. 2015;105(10): 877. http://dx.doi.org/10.7196/ SAMJNEW.8779

34. Aragão REM, Muccioli C, Barreira IMA, Ribeiro DCS, Timóteo CNG. Amaurose bilateral por menigoencefalite criptocócica: relato de caso. Arq Bras Oftalmol. 2008;71(1):101-3. http://dx.doi.org/10.1590/S000427492008000100021

35. Colombo AC, Rodrigues ML. Fungal colonization of the brain: anatomopathological aspects of neurological cryptococcosis. An Acad Bras Cienc. 2015;87(2):1293-1309. http://dx.doi.org/10.1590/00013765201520140704

36. Amaro MCO. Caracterização de isolados clínicos de Cryptococcus neoformans e Cryptococcus gattii quanto à susceptibilidade a fluconazol [dissertação]. Porto Alegre (RS): Universidade Federal do Rio Grande do Sul; 2006.

40. Freitas HF, Barros TF, Castilho MS. 2D chemometric studies of a series of azole derivatives active against fluconazole-resistant Cryptococcus gattii. J Braz Chem Soc. 2013;24(6):962-72. http://dx. doi.org/10.5935/01035053.20130122

41. Alves SH, Lopes JO, Costa JM, Klock C. Development of secondary resistance to fluconazole in Cryptococcus neoformans isolated from a patient with AIDS. Rev Inst Med Trop S Paulo. 1997;39(6):359-61. http://dx.doi. 
org/10.1590/S0036-46651997000600010

42. Franzot SP, Hamdan JS. In vitro susceptibilities of clinical and environmental isolates of Cryptococcus neoformans to five antifungal drugs. Antimicrob. Agents Chemother. 1996; 40(3): 822-824. http://dx.doi.org/10.1128/ AAC.40.3.822

43. Favalessa OC, Ribeiro LC, Tadano T, Fontes CJF, Dias FB, Coelho BPA, et al. Primeira descrição da caracterização fenotípica e susceptibilidade in vitro a drogas de leveduras do gênero Cryptococcus spp isoladas de pacientes HIV positivos e negativos, Estado de Mato Grosso. Rev Soc Bras Med Trop. 2009;42(6):661-65. http://dx.doi.org/1590/ S0037-86822009000600010

44. Kobayashi CCBA, Souza LKH, Fernandes OFL, Brito SCA, Silva AC, Sousa ED, et al. Characterization of Cryptococcus neoformans isolated from urban environmental sources in Goiânia, Goiás State, Brazil. Rev Inst Med Trop S Paulo. 2005;47(4):203-07. http://dx.doi.org/10.1590/S003646652005000400005

45. Silva PR, Rabelo RAS, Terra APS, Teixeira DNS. Suscetibilidade a antifúngicos de variedades de Cryptococcus neoformans isoladas de pacientes em hospital universitário. Rev Soc Bras Med Trop. 2008;41(2): 158-62. http://dx.doi.org/10.1590/S0037-86822008000200005

46. Alves SH, Boettcher CS, Oliveira DC, Tronco-Alves GR, Sgaria MA, Thadeu P, et al. Sporothrix schenckii associated with armadillo hunting in Southern Brazil: epidemiological and antifungal susceptibility profiles. Rev Soc Bras Med Trop. 2010;43(5):523-25. http://dx.doi.org/10.1590/ $\underline{\text { s0037-86822010000500010 }}$

47. Flores-García A, Sánchez-Gutiérrez R, VelascoRodríguez R, Zambrano-Parra A, Ruiz-Bernés S, Estrada CP. Interleucina-12 exógena evita disminución de actividad de mieloperoxidasa de macrófagos en gérbiles con esporotricosis. Rev Cubana Invest Bioméd. 2014;33(1):5260.

48. Morales LP, López MI, Cherta OQ, Rodríguez IR. Aislamiento microbiológico de Sporothrix Schenckii en un paciente inmunodeprimido. Presentación de un caso. Medisur. 2014;12(4):662-69.
49. Oliveira DC, Loreto ES, Mario DAN, Lopes PGM, Neves LV, Rocha MP, et al. Sporothrix schenckii complex: susceptibilities to combined antifungal agents and characterization of enzymatic profiles. Rev Inst Med Trop S Paulo. 2015;57(4):289-94. http://dx.doi.org/10.1590/ S0036-46652015000400003

50. Rosa CMG. Detecção de Aspergillus fumigatus em hemoculturas [dissertação de mestrado]. Porto: Universidade do Porto; 2008.

51. Poester VR, Klafke GB, Cabana AL, Adornes AC, Filho RPS, Xavier MO. Isolamento e identificação de fungos do gênero Aspergillus spp. de água utilizada na reabilitação de pinguins-de-magalhães. Ciênc Anim Bras. 2015;16(4):567-73. http://dx.doi.org/10.1590/1089$6891 \mathrm{v} 16 \mathrm{i} 428509$

52. Takahashi JP, Melhem MSC. Uso de fungicidas na agricultura e resistência a antifúngicos na clínica médica. Bol Inst Adolfo Lutz. 2014;24(1):40-1.

53. Alastruey-Izquierdo A, Melhem MSC, Bonfietti LX, Rodriguez-Tudela JL. Susceptibility test for fungi: clinical and laboratorial correlations in medical mycology. Rev Inst Med Trop S Paulo. 2015;57(19):57-64. http://dx.doi. org/10.1590/S0036-46652015000700011

54. Pontes L. Vigilância de resistência frente a antifúngicos azólicos de isolados clínicos de Aspergillus fumigatus [dissertação]. Campinas: Universidade Estadual de Campinas; 2018.

55. Braga VF. Determinação da prevalência das species, resistência a antifúngicos e perfil de virulência de Aspergillus spp. oriundos de amostras de origem clínica [dissertação]. Ribeirão Preto: Universidade de São Paulo; 2018. 